\title{
EDITORIAL
}

For reprint orders, please contact: reprints@futuremedicine.com

\section{Neoadjuvant chemotherapy for breast cancer: a role for short-term fasting?}

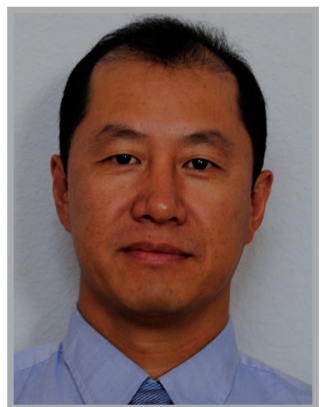

Jiaxin $\mathrm{Niu}^{* 1}$

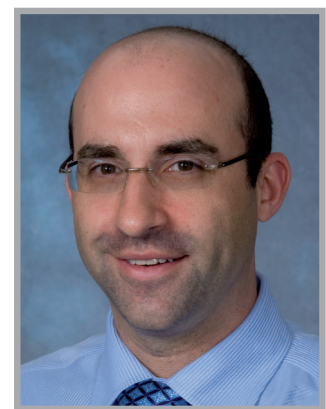

Glen J Weiss ${ }^{1}$
"Recent studies suggested that short-term fasting can protect normal mammalian cells, mice and possibly patients from the side effects of chemotherapy agents..."

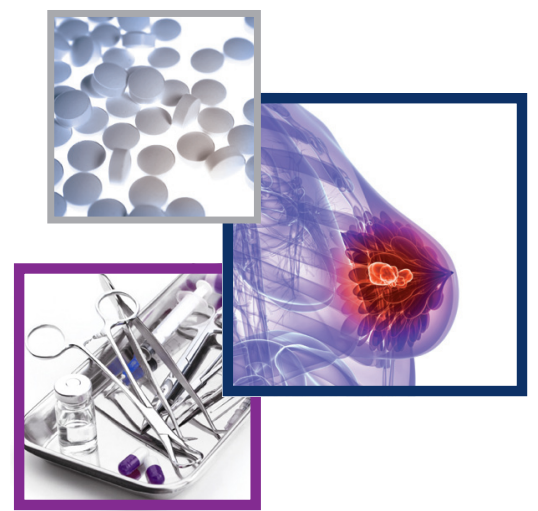

Breast cancer is the second leading cause of cancer death in women in the USA. An estimated 232,340 women will be diagnosed with breast cancer in 2013, with approximately $90 \%$ of women with breast cancer presenting with loco-regional disease [1]. Traditionally, most women undergo a definitive surgical procedure that allows for accurate staging followed by treatment plans consisting of systemic chemotherapy and radiation commensurate with staging and guideline recommendations.

Systemic chemotherapy is critical to eradicate occult micrometastatic disease after surgery. Adjuvant chemotherapy regimens have gone through rigorous head-tohead comparison in the past three decades, with anthracycline-taxane combinations regarded as the most active chemotherapy regimens for HER2-negative breast cancer [2]. Neoadjuvant chemotherapy has been established as a reasonable alternative for adjuvant chemotherapy, since multiple large randomized clinical trials have demonstrated that neoadjuvant chemotherapy provides identical disease-free (DFS) and overall survival (OS) benefits as adjuvant chemotherapy administered with the same regimens [3,4]. Neoadjuvant chemotherapy has also been shown to increase the breast conservation rate and decrease the rate of complete axillary lymph node dissection. Often, neoadjuvant chemotherapy represents the only reasonable option for women who present with more locally advanced disease and desire breast conservation. More importantly, response to neoadjuvant chemotherapy portends a good prognosis. Women with breast cancer that achieves a pathological complete remission (pCR) are expected to have excellent DFS and OS [5]. Irrespective of the different definitions of pCR in various clinical trials, the absence of invasive disease in both the breast and lymph nodes provides the best overall outcome. Evaluation of tumor responses to neoadjuvant regimens in patients who have not previously been exposed to any other systemic therapies could offer an efficient testing strategy to different regimens or interventions.

Unfortunately, up to $75 \%$ of women do not achieve pCR when they are treated with anthracycline-taxane-based neoadjuvant chemotherapy [5]. Among those with four to nine positive axillary lymph nodes

'Department of Medical Oncology, Western Regional Medical Center at Cancer Treatment Centers of America, 14200 W Celebrate Life Way, Goodyear, AZ 85338, USA

*Author for correspondence: Tel. +1 623207 3329; Fax. +1 623321 1528; jiaxin.niu@ctca-hope.com

"Despite the great strides we have made in the past few decades, there remains an unmet medical need for more effective chemotherapy." 
after neoadjuvant chemotherapy, 8-year DFS is only $40 \%$. Adding capecitabine and gemcitabine to anthracycline-taxane-based neoadjuvant chemotherapy does not improve clinical and pathological response rate, but rather increases the toxicity despite the suggestive evidence in the metastatic setting [6]. These results are in line with a few other adjuvant study results [7].

Despite the great strides we have made in the past few decades, there remains an unmet medical need for more effective chemotherapy. With limited resources, most oncologists believe the era to use large randomized and expensive trials to compare different adjuvant chemotherapy regimens is over. In contrast, neoadjuvant trials involve smaller sample size, less time and cost, providing an efficient trial design for assessing the efficacy of novel therapies. Several recent studies lend support that pCR is associated with improved DFS and OS, and could be used as a surrogate marker for DFS and OS in breast cancer patients [8]. In fact, with the accumulation of these data, a draft guidance released by the US FDA states that pCR to neoadjuvant treatment of highrisk early-stage breast cancer could be used as an end point to support accelerated drug approval. Unless we have a major breakthrough in novel therapy, one focus for future study design should be to enhance the efficacy of current well-proven neoadjuvant regimens without adding additional toxicity.

\section{Breast cancer \& metabolism}

Unlike their normal counterpart, cancer cells have long been known to exhibit various metabolic anomalies. Many cancer cells rely on aerobic glycolysis to maintain their proliferation regardless of the availability of oxygen - best known as the Warburg effect, first described in the 1920s [9]. Aberrant aerobic glycolysis is often accompanied by increased glucose uptake, which is the basis for the widely used 18 F-deoxyglucose PET imaging. After almost a century of biochemical research, our understanding of the mechanism involved in tumor metabolism is far from complete. Along these lines, clinical studies have associated host metabolism to cancer development, progression and prognosis. Indeed, epidemiologic research links high circulating glucose, insulin resistance and obesity with increased incidence of breast cancer [10]. Several clinical studies have convincingly demonstrated that hyperglycemia, insulin resistance and obesity confer a worse prognosis in early-stage breast cancer [11,12]. These same signaling molecules such as glucose, insulin or IGF are also involved in cancer cell metabolism, in particular, aerobic glycolysis. It seems that targeting these pathways or molecules is a rational next step in cancer therapy, and breast cancer provides a perfect clinical disease model for this kind of study. As a matter of fact, retrospective clinical studies have shown a reduction in cancer-related mortality in diabetic patients who take metformin, an antidiabetic drug [13]. Conversely, insulin use is associated with higher cancer-related death [14]. A retrospective study demonstrated that much higher pCR was achieved in diabetic patients with breast cancer who received both neoadjuvant chemotherapy and metformin than neoadjuvant chemotherapy alone [15]. Metformin has also been shown to be an inhibitor for IGF-1 receptor in preclinical studies - whether this effect has contributed to the finding in the breast cancer study is not known. Many prospective trials are currently ongoing to target cancer metabolism.

\section{Breast cancer \& short-term fasting}

Another rational approach is diet modification. The long-term $20-40 \%$ restriction in calorie intake is known as dietary restriction (DR). It may even take weeks to be effective, the resultant changes in glucose or IGF-1 levels tend to be modest and, thus, it would be highly unlikely to show any beneficial effect in the neoadjuvant or adjuvant setting. The expected weight loss from DR could worsen the pre-existent cachexia and likely further impair immune function and wound healing in metastatic cancer patients. The fact that DR has never been integrated in clinical cancer treatment after decades of preclinical studies reflects these practical concerns. Recent studies suggested that short-term fasting (STF) can protect normal mammalian cells, mice and possibly patients from the side effects of chemotherapy agents by reallocating energy toward maintenance pathways from reproduction and growth [16-19]. In contrast, cancer cells are unable to turn on this switch. Instead, constitutively active oncogenes continue to activate AKT or other pathways leading to an increase in oxidative stress and DNA damage [18]. This phenomenon is termed differential stress resistance, which is most likely mediated by inflammatory factors involving glucose, IGF-1 and other growth factors [17]. 
In animal models and retrospective cancer case series, STF appeared to decrease the side effects of chemotherapy (protective effects on normal cells) $[18,19]$. Interestingly, STF for $24 \mathrm{~h}$ before and after treatment with doxorubicin or cyclophosphamide (doxorubicin and cyclophosphamide combination is commonly used for breast cancer neoadjuvant chemotherapy) rendered 15 out of 17 cell lines (including two breast cancer cell lines) more sensitive to these drugs. STF for $48 \mathrm{~h}$ also demonstrated the ability to retard tumor growth of subcutaneous allografts of murine breast cancer cell line 4T1 or xenografts of triple-negative human breast cancer cell line MDA-MB-231 [20]. Furthermore, STF synergistically increased chemotherapy-induced DNA damage and prolonged the mice survival with metastatic murine breast cancer [20].

STF is inexpensive and readily available. In a small case series, ten cancer patients including four breast cancer patients volunteered to fast up to 4-6 days before and after chemotherapy, STF was deemed safe. With the accumulated preclinical evidence, it seems an opportune time to evaluate STF prospectively in the neoadjuvant breast cancer treatment setting. Currently, there are no clinical trials evaluating this clinical scenario; however, diet restriction and/or fasting is under clinical investigation in breast cancer and lymphoma receiving treatment [101-103]. Neoadjuvant chemotherapy offers an efficient and economic tool to study the safety and efficacy. By incorporating the feasibility and safety of STF in the neoadjuvant setting, we can determine if this could be a new advance towards mitigating toxicity with minimal cost. Once established, the clinical benefits can be further tested in the adjuvant and metastatic setting.

\section{Financial \& competing interests disclosure}

The authors have no relevant affiliations or financial involvement with any organization or entity with a financial interest in or financial conflict with the subject matter or materials discussed in the manuscript. This includes employment, consultancies, honoraria, stock ownership or options, expert testimony, grants or patents received or pending, or royalties.

No writing assistance was utilized in the production of this manuscript.

\section{References}

1 Siegel R, Naishadham D, Jemal A. Cancer statistics, 2013. CA Cancer J. Clin. 63, 11-30 (2013).

2 Dang C. The end of an era: shall we move forward? J. Clin. Oncol. 29, 3849-3851 (2011).

3 Fisher B, Brown A, Mamounas E et al. Effect of preoperative chemotherapy on local-regional disease in women with operable breast cancer: findings from National Surgical Adjuvant Breast and Bowel Project B-18. J. Clin. Oncol. 15, 2483-2493 (1997).

4 Bear HD, Anderson S, Brown A et al. The effect on tumor response of adding sequential preoperative docetaxel to preoperative doxorubicin and cyclophosphamide: preliminary results from National Surgical Adjuvant Breast and Bowel Project Protocol B-27. J. Clin. Oncol. 21, 4165-4174 (2003).

5 Rastogi P, Anderson SJ, Bear HD et al. Preoperative chemotherapy: updates of National Surgical Adjuvant Breast and Bowel Project Protocols B-18 and B-27. J. Clin. Oncol. 26, 778-785 (2008)

6 Bear HD, Tang G, Rastogi P et al. Bevacizumab added to neoadjuvant chemotherapy for breast cancer. N. Engl. J. Med. 366, 310-320 (2012).

7 Joensuu H, Kellokumpu-Lehtinen PL, Huovinen $\mathrm{R}$ et al. Adjuvant capecitabine, docetaxel, cyclophosphamide, and epirubicin for early breast cancer: final analysis of the randomized FinXX trial. J. Clin. Oncol. 30, 11-18 (2012).

8 von Minckwitz G, Untch M, Blohmer JU et al. Definition and impact of pathologic complete response on prognosis after neoadjuvant chemotherapy in various intrinsic breast cancer subtypes. J. Clin. Oncol. 30, 1796-1804 (2012).

9 Bayley JP, Devilee P. The Warburg effect in 2012. Curr. Opin. Oncol. 24, 62-67 (2012).

10 Bruning PF, Bonfrer JM, van Noord PA et al. Insulin resistance and breast-cancer risk. Int. J. Cancer 52, 511-516 (1992).

11 Goodwin PJ, Ennis M, Pritchard KI et al. Fasting insulin and outcome in early-stage breast cancer: results of a prospective cohort study. J. Clin. Oncol. 20, 42-51 (2002).

12 Goodwin PJ, Ennis M, Pritchard KI et al. Insulin- and obesity-related variables in early-stage breast cancer: correlations and time course of prognostic associations. J. Clin. Oncol. 30, 164-171 (2012).
13 Evans JM, Donnelly LA, Emslie-Smith AM et al. Metformin and reduced risk of cancer in diabetic patients. BMJ330, 1304-1305 (2005).

14 Bowker SL, Majumdar SR, Veugelers P et al. Increased cancer-related mortality for patients with Type 2 diabetes who use sulfonylureas or insulin. Diabetes Care 29, 254-258 (2006).

15 Jiralerspong S, Palla SL, Giordano SH et al. Metformin and pathologic complete responses to neoadjuvant chemotherapy in diabetic patients with breast cancer. J. Clin. Oncol. 27, 3297-3302 (2009).

16 Lee C, Longo VD. Fasting vs dietary restriction in cellular protection and cancer treatment: from model organisms to patients. Oncogene 30, 3305-3316 (2011).

17 Lee C, Safdie FM, Raffaghello L et al. Reduced levels of IGF-I mediate differential protection of normal and cancer cells in response to fasting and improve chemotherapeutic index. Cancer Res. 70, 1564-1572 (2010).

18 Raffaghello L, Lee C, Safdie FM et al. Starvation-dependent differential stress resistance protects normal but not cancer cells against high-dose chemotherapy. Proc. Natl Acad. Sci. USA 105, 8215-8220 (2008). 
19 Safdie FM, Dorff T, Quinn D et al. Fasting and cancer treatment in humans: a case series report. Aging (Albany NY) 1, 988-1007 (2009).

20 Lee C, Raffaghello L, Brandhorst S et al. Fasting cycles retard growth of tumors and sensitize a range of cancer cell types to chemotherapy. Sci. Transl. Med. 4, 124ra27 (2012).

\section{- Websites}

101 ClinicalTrials.gov. Effects of Short-term Fasting on Tolerance to Chemotherapy. http://clinicaltrials.gov/ct2/show/ NCT01304251?term =NCT+01304251 \&rank=1

102 ClinicalTrials.gov. Short-Term Fasting: Impact on Toxicity. http://clinicaltrials.gov/ct2/show/ NCT00936364?term=NCT00936364 \&rank=1

103 ClinicalTrials.gov. Short-Term Fasting Before Chemotherapy in Treating Patients With Cancer.

http://clinicaltrials.gov/ct2/show/ NCT01175837?term=NCT01175837 \&rank=1 